\title{
Sensing properties of asymmetric double-layer-covered tapered fibers
}

\author{
Francisco-Javier Bueno, Oscar Esteban, Natalia Díaz-Herrera, \\ María-Cruz Navarrete, and Agustín González-Cano
}

\begin{abstract}
A novel, to our knowledge, device based on a tapered optical fiber with a double-layer deposition including a metallic media is presented, and its properties are studied. The main novelty of the device consists of the introduction of a dielectric layer, whereas the systems depicted in the literature are simply metalcoated tapered fibers. The presence of the dielectric layer permits one to tune the response of the device to the refractive index of the surrounding medium. We have proved the suitability of this scheme for refractive-index sensing by depicting two measurement modes, namely, total power attenuation and spectral transmittance. (C) 2004 Optical Society of America

OCIS codes: $\quad 060.2370,240.6680$.
\end{abstract}

\section{Introduction}

Optical excitation of surface plasma waves has been widely used in many optical fiber sensor configurations in the past years. One of the most reported configurations consists of a multilayer, including metallic media, ${ }^{1-4}$ deposed onto a side-polished fiber (D fiber). Because surface plasma waves supported by a planar metal layer are transverse-magnetic (TM) polarized, these devices have shown a polarizationdependent response. As the parameters of the multilayer are fixed, the final response of devices depends on the refractive index of the medium surrounding the structure. When the incident light is TM polarized, a surface plasmon mode can eventually be excited for a specific refractive index of the outer medium, and the attenuation of the transmitted light occurs. This attenuation is the measurable magnitude and can be compared with the total light

F.-J. Bueno is with the Departamento Teoría de la Señal, Escuela Politécnica, Universidad de Alcalá, 28871 Alcalá de Henares, Madrid, Spain. Ó. Esteban (oscar@depecu.uah.es) is with the Departamento de Electrónica, Escuela Politécnica, Universidad de Alcalá. N. Díaz-Herrera and M.-C. Navarrete are with the Departamento de Óptica, Facultad de Ciencias Fisicas, Universidad Complutense de Madrid, Ciudad Universitaria s/n, 28040 Madrid, Spain. A. González-Cano is with the Departamento de Óptica, Escuela Universitaria de Óptica, Universidad Complutense de Madrid, Arcos de Jalón s/n, 28037 Madrid, Spain.

Received 27 June 2003; revised manuscript received 9 October 2003; accepted 16 December 2003.

0003-6935/04/081615-06\$15.00/0

(C) 2004 Optical Society of America launched into the fiber. The final result is a dip in the curve of the total transmitted power as a function of outer refractive index.

Optical fiber devices based on tapered fibers have also been recently reported. ${ }^{5-7}$ These devices, in which both cladding and core diameters are reduced to reach the evanescent field, are coated with a metal layer that can support different surface plasma modes. ${ }^{8,9}$ These devices are suitable in two configurations, either symmetric or asymmetric, depending on whether either the whole circumference or only one side of the tapered fiber is coated. The first one presents the advantage of the polarization-independent response due to the symmetry of the complete device, whereas the second configuration keeps a polarizationdependent response like the side-polished-based sensors because of the lack of such symmetry. When the geometrical parameters are established, a characteristic transmittance spectrum is obtained for a metal layer in a range of outer refractive indices.

Devices reported until now use only a single metal cover, in either a symmetric or an asymmetric configuration. In this paper we have added a second dielectric layer over the metallic one to obtain surface-plasma-mode resonances at shorter wavelengths at a lower refractive-index range. We have focused our attention in asymmetric devices because it is easier to depose the layers in this way, so the production of these devices is faster and cheaper. Also, asymmetric devices permit us to characterize, in a more complete way, the polarization dependence of this kind of element.

The devices reported here are based on the mea- 
surement of the transmission properties of light guided by the optical fiber. The output signal can be processed in two ways, taking into account the total power transmitted through the device as usual in D-fiber sensors or checking the spectral properties of such transmitted light.

In this paper we present some experimental results in both cases, which allow us to obtain a complete characterization of the behavior of these devices with respect to the changes of all the parameters of tapered fibers and the media surrounding them.

\section{Operational Principle}

The physical principle in which these devices are based is the surface-plasma-wave excitation by the evanescent field of the optical fiber. This evanescent field can be reached by the narrowing of the fiber's cross section in the tapered region. The simplest theoretical treatment of this problem involves a dielectric cylinder covered with a metallic shell. This model can be directly applied to the case in which a tapered fiber is metal coated and surrounded by another dielectric medium. The analysis of this problem by use of a standard boundary-value method shows that we can find two surface plasma modes for each azimuthal order. ${ }^{5-9}$ When the effective refractive index of the surface plasma modes approaches the value of the effective refractive index of the modes of an uncoated tapered fiber, resonant coupling between the modes with the same azimuthal order of both substructures can be observed at a specific combination of geometrical and material parameters, and a resonant dip is observed in the transmission spectrum.

The lack of cylindrical symmetry implies that the transmission spectra depend on the polarization of the input light. The incident mode of the fiber, which is a hybrid mode with azimuthal order $m=1$, can excite surface plasma modes with azimuthal order $m \neq 1$ since they are no longer orthogonal. ${ }^{7}$ Consequently, the transmission spectra of these devices show more than one dip, and we will record different spectra for either transverse-electric (TE) or TM polarization of the input light.

A specific geometrical configuration (taper waist diameter and layer thickness) provides a characteristic transmittance spectrum for either TE or TM polarization of the input optical field. The addition of a second dielectric layer causes the displacement of the complete spectrum toward shorter wavelengths. This displacement provides us a method of selecting a wavelength range to perform our measurements and permits us to obtain different application points because the spectrum dips appear for lower refractive indices of the outer medium.

These devices can operate in two ways: (1) as a frequency-output mode in which the transmission spectra move toward longer wavelengths as the outer refractive index of the dielectric medium surrounding

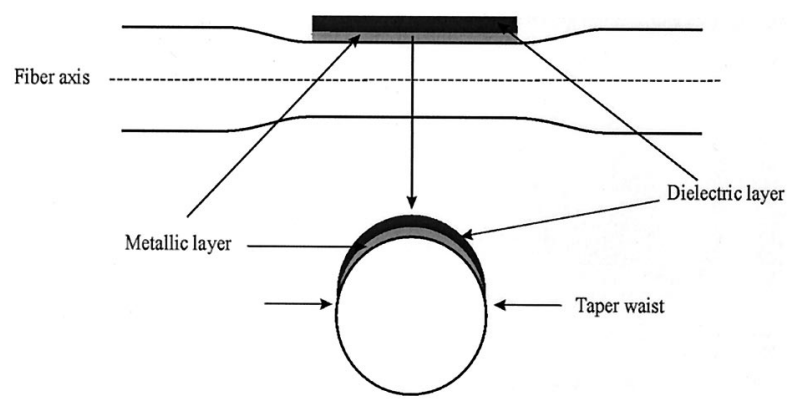

Fig. 1. Cross section and side view of the asymmetric device. The double-layer deposition over the uniform waist is shown.

the structure increases or (2) as a power-output mode in which the total optical power transmitted by the device changes with the outer refractive index, as is usual in D-fiber-based devices. ${ }^{1-4}$

\section{Sensor Fabrication and Experimental Setup}

The process of the device fabrication can be separated into two steps. The first one is to decrease the fiber diameter so that we can reach the evanescent field of the mode propagated by the unperturbed fiber. We follow the procedure developed by Kenny et al., ${ }^{10}$ the so-called traveling burner. This technique has been widely used by different authors ${ }^{5-7,11,12}$ to make a huge variety of devices based on tapered optical fibers. With this procedure, we obtain different substrates with different uniform waists of tapered fiber over a length of several millimeters with low loss.

The optical fiber we use is a conventional singlemode step-index fiber at a nominal wavelength of 820 $\mathrm{nm}$. The waists of the different devices we have made are in the $30-40-\mu \mathrm{m}$ range, and the losses of the tapers are less than $0.3 \mathrm{~dB}$.

The second step of the production process consists of the deposition of layers on the substrates obtained in the first step explained above. The deposition method is physical vapor deposition based on the procedure depicted by Alonso et al. ${ }^{1}$ The asymmetric device is thus obtained by deposition of a double layer on one side of the tapered fiber. A schematic view of the complete device is shown in Fig. 1.

The values of the thickness of materials deposited vary from zero at the edges to a maximum value at the top of the waist. We have chosen a first layer of aluminium 8-nm thick and a second layer of titanium dioxide 60-nm thick. This choice of thickness and materials is a consequence of our previous papers, ${ }^{3,4}$ in which D-type fibers with a double metallicdielectric layer were used as refractive-index sensors, and permits us to make measurements in the 780880-nm wavelength range for refractive indices of the outer medium between 1.32 and 1.41 .

We can test the behavior of these devices in two ways, as we said before. These two ways demand different light sources and detectors. If we want to make any power-output-based measurement, we need a powerful light source. On the other hand, if we want a frequency-output-based measurement, we 


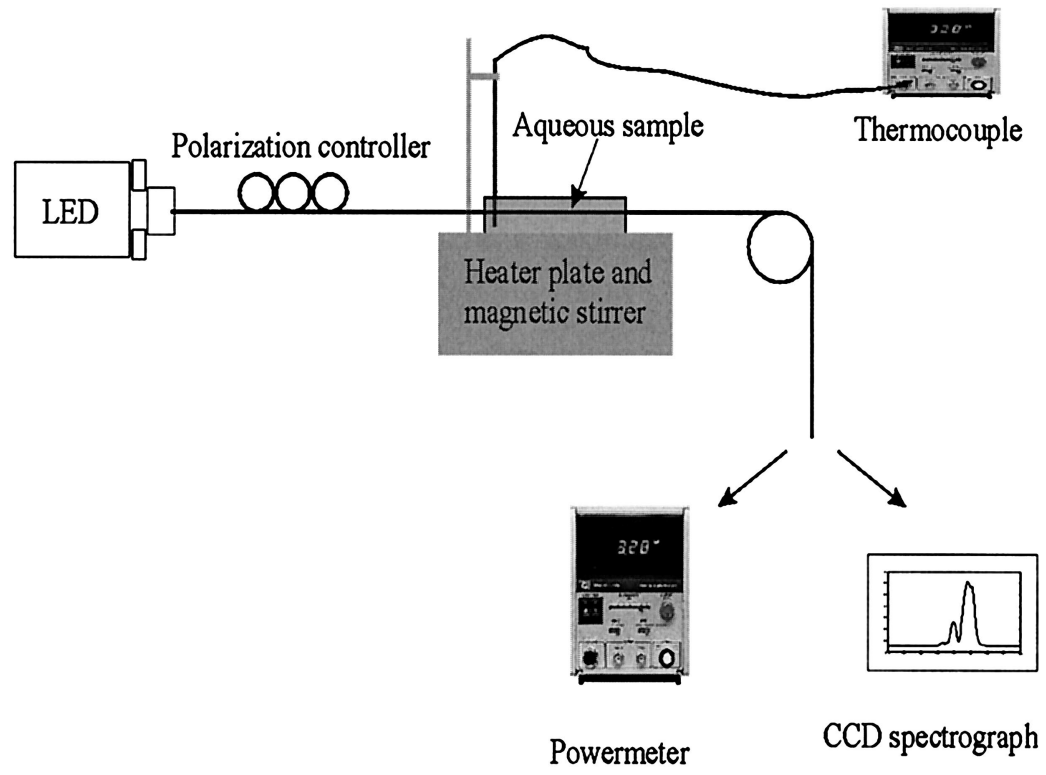

Fig. 2. Schematic view of the setup.

need a wide-spectrum light source and a spectrometer as a detector. In both cases, polarization control of the light source is required because the final response of the device depends on the polarization of the input field, as is explained in Section 2.

To simplify the setup, we have chosen a light source that is powerful enough to carry on the poweroutput measurements with a simple photodiode detector and has a spectral width wide enough to obtain several dips in the transmitted spectra when the frequency-output test is performed. The experimental setup used for the device characterization is shown schematically in Fig. 2, in which light is provided by a commercial LED with a peak wavelength at 830-nm and a 50-nm spectral half-width; we only have to change between a powermeter and a CCD spectrograph to perform both tests. As is mentioned above, the lack of symmetry of the device implies that it reacts to both polarization states of the incident light. For that reason, an in-line optical fiber polarizer $^{1}$ is used to polarize linearly the output light from the LED, and a set of Lèfevre loops is used to control the polarization plane of the resultant linearly polarized light when required. Each polarization state of the input light shows a different transmission spectrum through the device, with different dip amplitudes in a given spectral range, so we use the polarization controller to obtain the maximum contrast between dips in our operational range without strictly choosing either TE or TM polarization.

Both kinds of measurement are referred to the transmitted light through the device when the active area is in contact with an outer medium, which is an aqueous media with variable refractive index in our case. Variations of the refractive index of the outer media are achieved by use of a mixture of distilled water and chemically pure ethylene glycol; the refrac- tive index of the solution depends on the concentration of ethylene glycol according with the empirical law ${ }^{1}$

$$
n(T)=n_{\mathrm{H}_{2} \mathrm{O}}(T)+0.111 \frac{V_{\text {eth }}}{V_{\text {tot }}},
$$

where $n$ is the refractive index of the mixture, $n_{\mathrm{H}_{2} \mathrm{O}}$ is the refractive index of pure water, $V_{\text {eth }}$ is the volume of ethylene glycol, and $V_{\text {tot }}$ is the total volume of the mixture. Both refractive indices are dependent on temperature, and this fact is taken into account in the calculation. A wide range of refractive indices from 1.327 (approximately pure water) to 1.41 can be covered with this method.

\section{Experimental Results}

\section{A. Power-Output Mode}

The first tests performed with our devices were made following the same procedure used with the D-fiber configuration. ${ }^{3}$ The transmitted power is measured for each value of refractive index of the outer medium and normalized to the value of transmitted power when the outer medium is air $(n \approx 1)$.

Given that the devices respond to both polarization states, we made a first set of measurements with unpolarized light by directly launching the light of the LED into the fiber, and we measured the total power transmitted through the device. The optical resonance of surface plasma modes produces the attenuation in the transmitted power, which can be measured. The transducer element was made with the layers already mentioned in Section 3. Three different taper waist diameters were tested to evaluate their influence on the final response of the device. The results of these tests can be seen in Fig. 3, in which relative transmittance is used for comparison purposes. As can be seen, the complete set of 


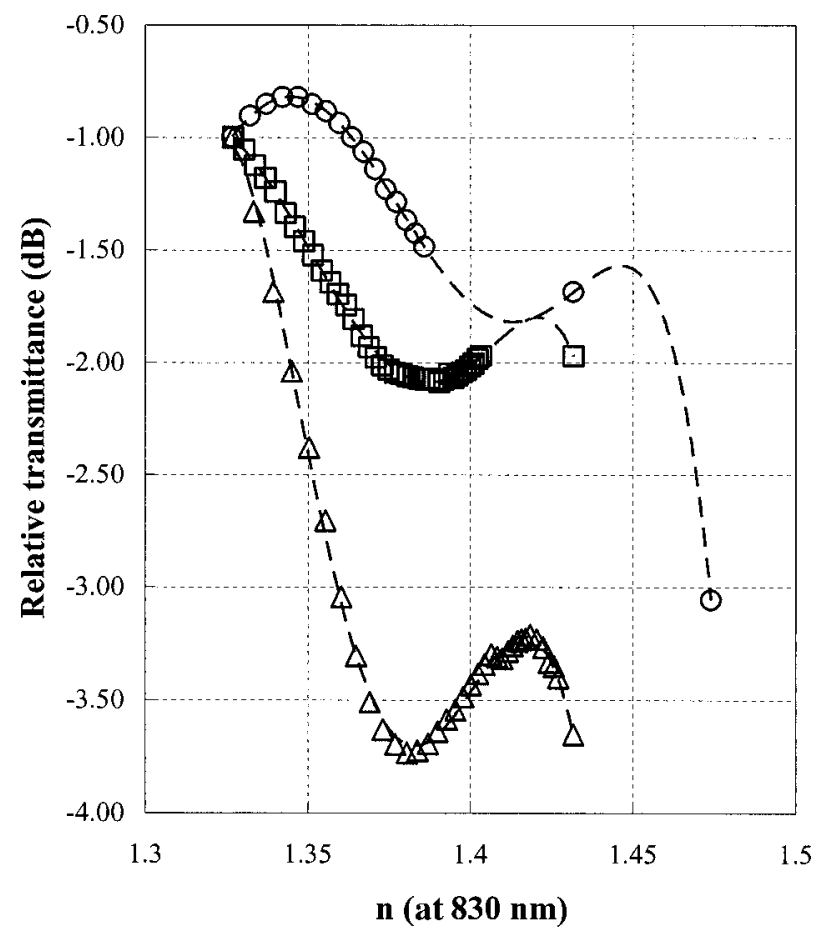

Fig. 3. Effect of the waist diameter on the relative transmittance of the device for unpolarized light. The figure has been arranged in order that all measurements start at the same value of transmittance only for comparison purposes. Circles, squares, and triangles are used for waist diameters of 40,37 , and $35 \mu \mathrm{m}$.

acquired data in each case moves toward lower refractive indices of the outer medium as the taper waist diameter decreases. Simultaneously, sensitivity of the device with respect to variations in the outer refractive index increases as the taper waist decreases if we observe the linear part of the plotted results.

We performed a second test with this kind of output by employing only TM-polarized input light. The in-line optical fiber polarizer and a set of Lèfevre loops were used for this purpose. It must be noted that this configuration requires that the fiber between the polarizer and the transducer be kept straight to preserve the polarization plane along the path. With this setup, the overall power transmitted in the case of air as the outer medium decreases, and the transmitted power changes with the outer refractive indices as in the previous case. The result of this test is compared with the previous case in Fig. 4 for a device with a taper waist diameter of $37 \mu \mathrm{m}$.

We can observe in Fig. 4 how the sensitivity increases by using polarized light instead of unpolarized light and how the same operational range, in which the response is almost linear, is kept. Only a slight displacement in the minimum transmittance is observed.

From Fig. 3 and 4, we see that with these devices we can select the operational range by using different materials and thicknesses, as we can do in D-fiber

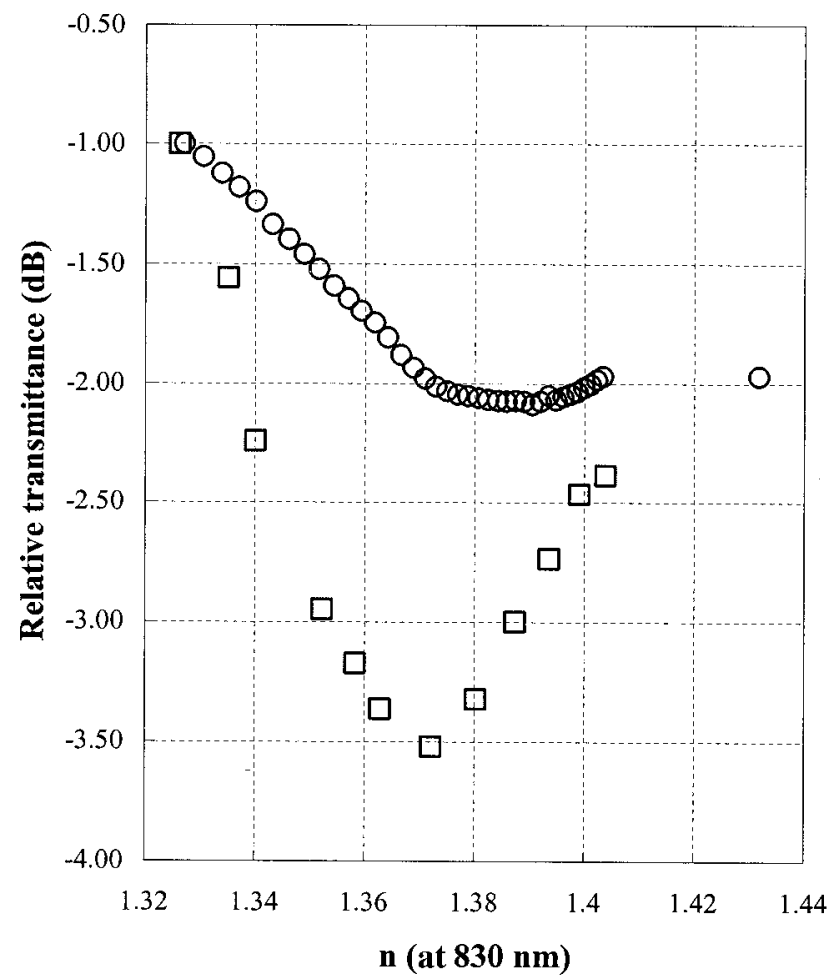

Fig. 4. Relative transmittance of the devices, with (circles) and without (squares) polarization control. Better sensitivity is achieved for TM-polarized light.

configurations, or by changing the taper waist diameter, which provides us a new parameter to select our application point.

\section{B. Frequency-Output Mode}

Another type of measurement we can make are spectral ones. For these measurements, we need only to replace the powermeter with a CCD spectrograph and observe the spectral properties of transmitted light through the device. The spectral range is limited by the width of the light source, in our case, the mentioned LED. The procedure followed here is almost the same as that explained above. We obtain the spectral transmittance for different refractive indices of the outer medium. As the dips obtained for TE and TM polarizations are given for a fixed structure, we only use the polarization control to obtain the maximum contrast between maxima and minima in the spectral transmittance, as shown in Fig. 5 in which the transmittance of a device with a $37-\mu \mathrm{m}$ waist diameter of tapered fiber and with an aluminium layer $8 \mathrm{~nm}$ thick and a titanium dioxide layer $60 \mathrm{~nm}$ thick is plotted for outer refractive indices of 1.345 (solid curve) and 1.366 (dashed curve).

From Fig. 5, we can select a dip in the transmittance spectrum and track its displacement with the refractive index of the outer medium. As in the case of power-output measurements, we can select the operational point inside the complete spectrum by an appropriate choice of materials and thickness and 


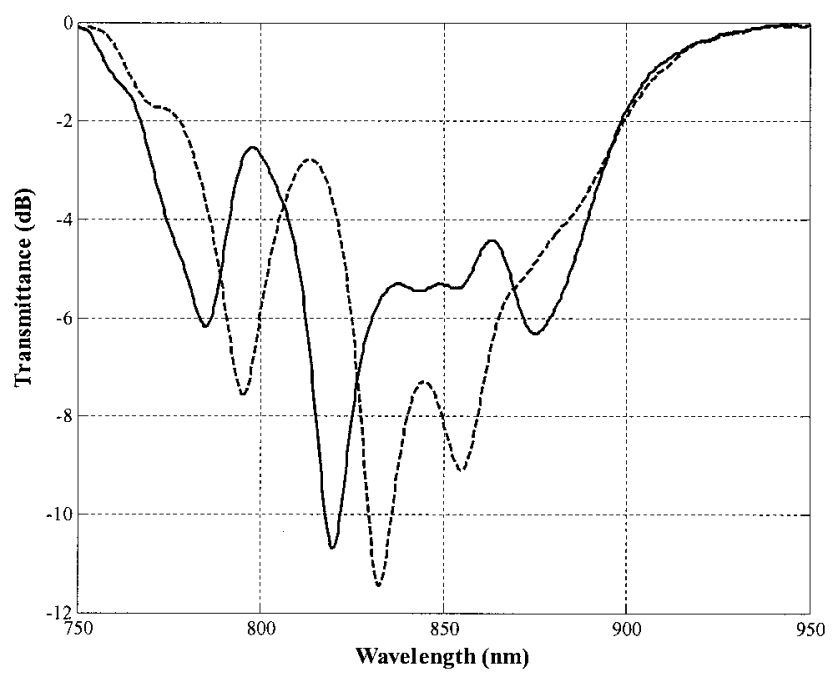

Fig. 5. Spectral transmittance of a device with taper waist diameter of $37 \mu \mathrm{m}$ and for outer refractive indices of 1.345 (solid curve), and 1.366 (dashed curve).

also by either increasing or decreasing the waist diameter. In Fig. 6 experimental results are shown for the displacement obtained in a minimum of transmittance with the outer refractive indices and for two waist diameters-circles for $31 \mu \mathrm{m}$ and squares for $37 \mu \mathrm{m}$. In the figure we can see how the sensitivity is almost the same in both cases, but the position of

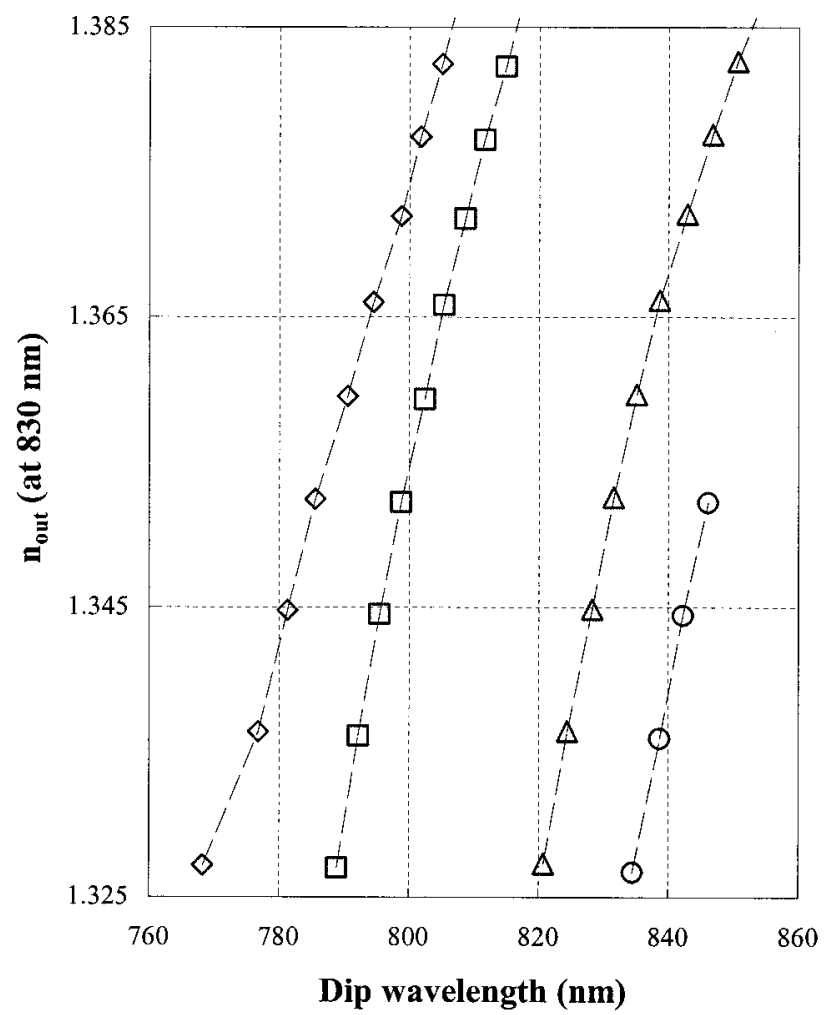

Fig. 6. Displacement of a selected transmittance dip by the variation of the outer refractive index for different waist diameters: $40 \mu \mathrm{m}$ (diamonds), $37 \mu \mathrm{m}$ (squares), $35 \mu \mathrm{m}$ (triangles), and $31 \mu \mathrm{m}$ (circles). minimum wavelengths are displaced to higher values as the waist diameter increases.

\section{Analysis of the Results}

The results presented, in both modes of measurement, confirm the possibility of excitation of surface plasma waves in the proposed structure. In that the two types of measurement are intrinsically different, we obtain different information, and both measurements can be used as operating principles for refractive-index sensors. We analyze now, briefly, some specific facts of the obtained results.

One significant feature of this sensor is the appearance of several dips in the spectral transmittance curves. This may be explained as follows: Because we deposit the layers only in part of the cylindrical section of the fiber, this deposition does not provide us with a uniform thickness, and we can consider that the structure is really equivalent to a set of different fiber-multilayer substructures, each of them having different geometrical parameters. This fact is important in that, as stated in Section 2, the excitation of a surface plasma wave is dependent on the parameters of the structure, as well as on the wavelength used. The existence of a local variation of the thickness in the deposited region implies that the conditions for the excitation of surface plasma waves can be fulfilled locally within the sensing area for different combinations of wavelength and local thickness for the same refractive index of the outer medium. So to speak, we can excite different surface plasma waves along the complete length of fiber where the evanescent field can reach the deposited double layer (including a part of the transition regions of the tapered fiber). As the refractive index of the outer medium changes, the multilayer structure also changes, and another set of surface plasma waves are excited as can be seen in Fig. 5 , in which the complete transmission spectrum moves toward higher wavelengths as the outer refractive index increases.

Also, because the spectral width of the LED source is finite, resonance dips with different intensities in the transmitted spectra appear and disappear at the left and right sides, respectively, as the outer refractive index increases. This fact is also reflected in the output-power mode in that the measurements imply the integration of the energy carried by the complete spectrum.

\section{Conclusions}

We have studied the properties of a new kind of device, namely, a tapered optical fiber covered with a double layer (metal plus dielectric). These devices can operate as refractive-index sensors by surface plasmon resonance in the metal layer produced by the evanescent coupling with the field guided in the tapered region. The presence of the dielectric layer makes it possible to tune the operating region of the sensor. Tapers with this second layer have not been reported in the literature, to our knowledge. In this 
way, the characterization that we perform in terms of dependence with the refractive index in the surrounding medium is intrinsically interesting and justifies the suitability of these devices as optical fiber sensors for measuring magnitudes whose variations affect the refractive index.

We have used asymmetric devices and considered two measuring modes, namely, total power and spectral transmittance. In both cases the dependence is clear and can be conveniently explained by our invoking the excitation of surface plasmons in the metal layer. We have also studied the influence of the diameter of the taper waist in that this parameter is a novel degree of freedom that also can be used to select the operating point of the devices. We have checked the influence of polarization in the behavior of the devices and shown that, in principle, the sensors can work with or without polarization control.

We can conclude that these devices are technologically feasible, versatile, and well adapted for their use as sensors. The use of a LED illumination source and a compact, simple setup are also important features in that sense.

We thank E. Bernabeu for his valuables comments and suggestions and C. Cosculluela from the Departamento de Física Aplicada of the Universidad de Zaragoza, Spain, for helping us with the devices' elaboration. This research has been partially supported by European Union project Multiparametric In Situ Spectroscopic Measuring System for Coastal Monitoring, contract EVK3-CT2000-00519, and Spanish project Aplicación de sensores de fibra óptica al control in-situ de parámetros físicos el medio acuático, Programa Nacional de Recursos Naturales, Ministerio de Ciencia y Tecnología, ref. REN 2001-1495.

\section{References}

1. R. Alonso, F. Villuendas, J. Tornos, and J. Pelayo, "New in-line optical-fiber sensor based on surface plasmon excitation," Sens. Actuators A 37-38, 187-192 (1993).

2. R. Alonso, J. Subias, J. Pelayo, F. Villuendas, and F. Tornos, "Single-mode, optical-fiber sensors and tunable wavelength filters based on the resonant excitation of metal-clad modes," Appl. Opt. 33, 5197-5201 (1994).

3. Ó. Esteban, M. C. Navarrete, A. González-Cano, and E. Bernabeu, "Measurement of the degree of salinity of water with a fiber-optic sensor," Appl. Opt. 38, 5267-5271 (1999).

4. Ó. Esteban, M. C. Navarrete, A. González-Cano, and E. Bernabeu, "Simple model of compound waveguide structures used as fiber-optic sensors," Opt. Lasers Eng. 33, 219-233 (2000).

5. A. Díez, M. V. Andrés, and D. O. Culverhouse, "In-line polarizers and filters made of metal-coated tapered fibers: resonant excitation of surface plasma modes," IEEE Photon. Technol. Lett. 10, 833-835 (1998).

6. A. Díez, M. V. Andrés, and J. L. Cruz, "Hybrid surface plasma modes in circular metal-coated tapered fibers," J. Opt. Soc. Am. A 16, 2978-2982 (1999).

7. A. Díez, M. V. Andrés, and J. L. Cruz, "In-line fiber-optic sensors based on the excitation of surface plasma modes in metalcoated tapered fibers," Sens. Actuators B 73, 95-99 (2001).

8. S. J. Al-Bader and M. Imtaar, "Optical fiber hybrid-surface plasmon polaritons,” J. Opt. Soc. Am. A 10, 83-88 (1993).

9. S. J. Al-Bader and M. Imtaar, "Azimuthally uniform surfaceplasma modes in thin metallic cylindrical shells," IEEE J. Quantum Electron. 28, 525-533 (1992).

10. R. K. Kenny, T. A. Birks, and K. P. Oakley, "Control of optical fiber taper shape," Electron. Lett. 77, 1654-1656 (1991).

11. T. A. Birks, P. St. J. Russell, and C. N. Pannell, "Low power acousto-optic device based on a tapered single-mode fiber," IEEE Photon. Technol. Lett. 6, 725-727 (1994).

12. D. O. Culverhouse, T. A. Birks, S. G. Farwell, and P. St. J. Russell, "3 33 all-fiber routing switch," IEEE Photon. Technol. Lett. 9, 333-335 (1997). 